\title{
Detection of Spontaneous Vesicle Release at Individual Synapses Using Multiple Wavelets in a CWT-Based Algorithm
}

\author{
Stefan Sokoll ${ }^{1,2}$, Klaus Tönnies ${ }^{2}$, and Martin Heine ${ }^{1}$ \\ 1 Group Molecular Physiology, Leibniz Inst. for Neurobiology, Magdeburg, Germany \\ 2 Dept. of Simulation and Graphics, Otto-von-Guericke Univ., Magdeburg, Germany
}

\begin{abstract}
In this paper we present an algorithm for the detection of spontaneous activity at individual synapses in microscopy images. By employing the optical marker pHluorin, we are able to visualize synaptic vesicle release with a spatial resolution in the $\mathrm{nm}$ range in a non-invasive manner. We compute individual synaptic signals from automatically segmented regions of interest and detect peaks that represent synaptic activity using a continuous wavelet transform based algorithm. As opposed to standard peak detection algorithms, we employ multiple wavelets to match all relevant features of the peak. We evaluate our multiple wavelet algorithm (MWA) on real data and assess the performance on synthetic data over a wide range of signal-to-noise ratios.
\end{abstract}

Keywords: multiple wavelets, continuous wavelet transform, peak detection, pHluorin, individual synaptic activity, microscopy.

\section{Introduction}

Neuronal signal transmission relies on the release of neurotransmitters from vesicles at chemical synapses. Efficacy of synaptic transmission is highly variable, and thereby participating in controlling the information flow in the brain. Therefore, the estimation of biophysical parameters modulating transmission efficacy is of great interest for neurobiologists.

Due to the nanometer-sized structures of chemical synapses, optical methods based on fluorescence microscopy are preferable for the analysis of neurotransmitter release. The most direct method uses $\mathrm{pH}$-sensitive variants of green fluorescent protein (pHluorins) coupled to the inside of synaptic vesicles [1. As depicted in Fig. 1 pHluorins change their fluorescence during vesicle recycling due to the $\mathrm{pH}$ gradient between the in- and outside of vesicles. These changes can be detected and have been used to analyze the kinetics of endocytosis [2], the quantification of different vesicle release pools [3] and involved molecules [4. However, the computational procedures to detect activity still require manual interaction. Neurobiologists evoke single action potentials, that may lead to vesicle fusion, by image-locked electrical stimulation. Small regions with infocus synapses are selected and difference images at the time of stimulation are calculated. 


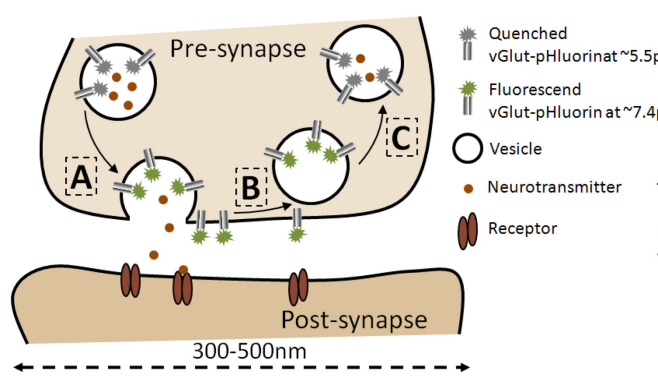

(a)

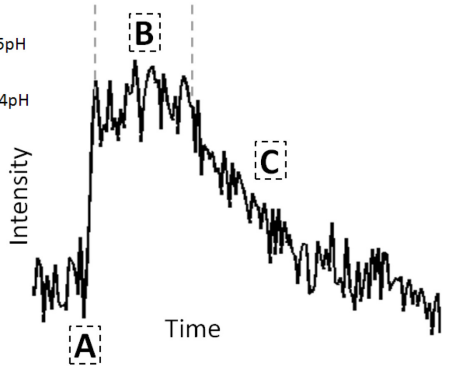

(b)

Fig. 1. Neuronal signal transmission at chemical synapses (a). At the low pH inside vesicles, pHluorins are quenched and thus hardly fluorescent. Upon vesicle fusion with the pre-synaptic membrane (exocytosis), pHluorins undergo a conformational change and their fluorescence increases (A). At the beginning of endocytosis, vesicles get recycled (B) and reacidify (C), thus, pHluorins get quenched again. The resulting intensity signal (b) is characterized by an immediate increase of intensity (A), followed by a dwell until endocytosis (B) and an intensity decay due to vesicle reacidification (C).

To our knowledge, there is no automated approach for the detection of individual synaptic communication. We present an algorithm based on the continuous wavelet transform (CWT) that also detects spontaneous events of active synapses 1 . This is particularly important for the analysis of changes in transmission efficacy and must be robust to varying signal strengths due to off-focus synapses and variations in the number of released vesicles per action potential.

\section{Related Work}

Peak detection in one-dimensional signals is challenging due to measurement noise and the lack of unique discriminating features. The most basic method is amplitude thresholding. Template matching is more robust with respect to noise since it also analyzes the shape of the peak. For a known shape, matched filtering [5] provides optimal detection under Gaussian noise. If only estimates are available, thresholding can be performed on similarity measures of the signal 6 . However, performance decreases if the peaks significantly vary in shape or size.

On the other hand, algorithms that use no prior shape information and perform blind equalization instead [7,8, require sufficiently large amounts of data and are highly sensitive to artifacts. Assuming that basic peak properties are preserved over all peaks, the wavelet transform is another alternative. Here, the signal is correlated with a mother wavelet at different scales, which enhances peak properties in the wavelet coefficients. Thresholding can then be applied on sets of wavelet coefficients 911 .

\footnotetext{
1 The software, including a GUI, is available at http://sourceforge.net/projects/ isad/
} 
A major drawback of CWT-based analysis is the usage of a single, probably suboptimal, wavelet for all peaks. We, therefore, propose the combination of multiple wavelets in a CWT-based algorithm referred to as MWA to match shape properties that cannot be addressed by a single wavelet.

\section{Method}

Our MWA peak detection method operates on intensity responses of individual synapses in microscopy images. Active synapses appear as elliptic spots in the mean intensity image of all acquired pHluorin time lapse images and are detected using the top hat filter. These candidates are fitted with an elliptical Gaussian function using a restricted region around synapses. The support regions are computed using an inverse watershed algorithm. This allows for the separation of nearby synapses and application of constraints on the size of synapses. Furthermore, we co-transfect any culture with synapsinRFP, which serves as a marker for the pre-synapse and include only those synapses that are double positive for pHluorin and synapsin. The individual intensity response for each point in time is then computed from the mean intensities within a fixed region centered over each synapse (see Fig. 2a). The region must be as small as possible to optimize the signal-to-noise ratio (SNR) but large enough to contain both sites of vesicle recycling. Experiments show that both requirements are robustly fulfilled taking a diameter of three times the size of the point spread function of the microscope. The wavelet transform decomposes a signal $s(t)$ into shifted and scaled versions $\psi_{a, b}(t)$ of a mother wavelet $\psi(t)$ to project it into the wavelet space:

$$
C(a, b)=\int_{\mathbb{R}} s(t) \psi_{a, b}(t) d t, \psi_{a, b}(t)=\frac{1}{\sqrt{a}} \psi\left(\frac{t-b}{a}\right) a, b \in \mathbb{R}, a>0 .
$$

The wavelet coefficients $C(a, b)$ determine the similarity between the signal and the mother wavelet at different scales $a$ and translations $b$.

The immediate increase of intensity, the variation in the dwell time, and the intensity decay are the characterizing peak shape features even though the kinetics are superimposed by photobleaching. For successful CWT-based peak detection, the mother wavelet must match the basic peak shape. Unfortunately, the major wavelet families provide no wavelet that supports all features. We, therefore, propose to cover the peak features by different wavelets and fuse their information after processing in the wavelet space. By visual inspection, we find that a combination of the Haar and the Bior3.1 wavelet describes the major peak shape characteristics (see Fig. $2 \mathrm{~b}$ ). Here, the Haar wavelet detects the intensity increase in a small support region and Bior3.1 adjusts to the dwell time, limited by the local discontinuities at the intensity increase and the onset of the decay. The actual peak detection method basically comprises six steps that are illustrated in Fig. 3 using a section from signal A in Fig. 2a. In step I, the signal is projected twice into the wavelet space using the two wavelets independently and resulting in $C_{\text {haar }}$ and $C_{\text {bior }}$. The projections are directly applied on the signal without prior baseline removal as the influence of photobleaching is minor and monotonically decreasing in the peak support region [11]. 

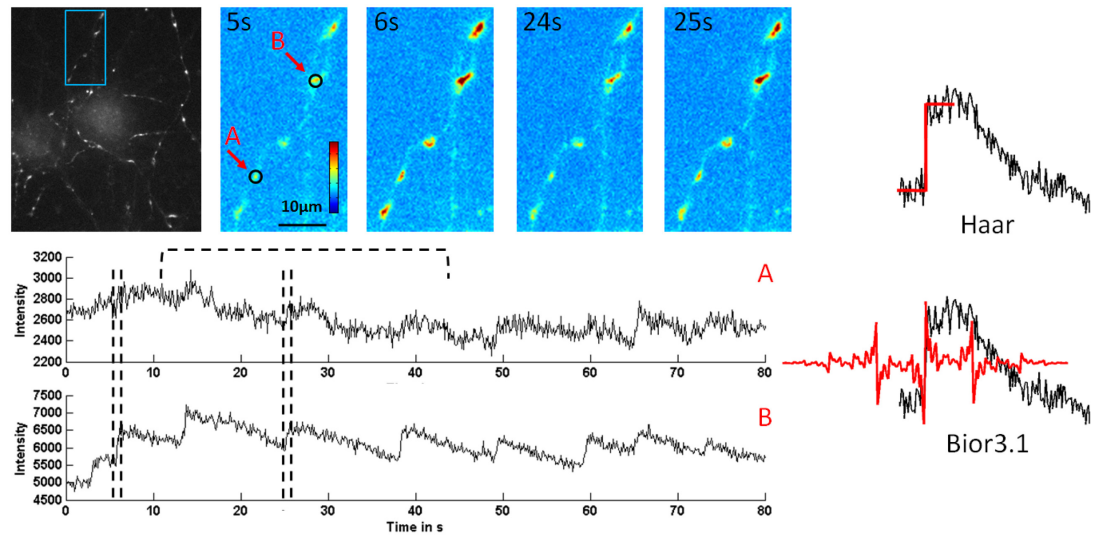

A

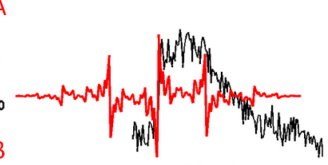

Bior3.1

(a)

(b)

Fig. 2. The change in intensity during synaptic activity is highlighted using a false colored region of the microscopy image (a). Below, the two intensity signals illustrate that synapses in the same image can have different SNR and baseline intensity. (b) depicts the use of the two wavelets, presenting the decomposing wavelet functions $\psi$ in red and an almost ideal intensity response in black. The wavelets are mirrored at the $\mathrm{x}$ axis for illustration.

In step II, the most significant coefficients are selected using the thresholding strategy, proposed for wavelet denoising by Donoho and Johnstone [12]:

$$
C_{t}(a, b)=\left\{\begin{array}{ll}
C(a, b) & \text { if } C(a, b) \geq t_{a} \\
0 & \text { otherwise }
\end{array} .\right.
$$

The threshold $t_{a}$ for each scale $a$ is derived from the noise coefficients at that scale. As they are unknown, a reasonably unbiased estimate can be obtained from the median absolute deviation $\tilde{\sigma}_{a}$, giving $t_{a}=\tilde{\sigma}_{a} / 0.6745$ [13]. If the variation in peak size is known from biophysical properties, then the relevant coefficients can also be restricted from the support range of the mother wavelet [9, 10]. In our application, the current dwell time of a peak is unknown but follows an exponential distribution with a mean lifetime of $\sim 14 \mathrm{~s}[2$. For any point in time we, therefore, continuously select all remaining coefficients up to the scale $a_{\text {dwell }}(b)$ that has the highest coefficient $\hat{c}(b)$, or is a defined maximal scale $\hat{a}$. This selects the most useful scales for a peak, as significant signal structures propagate across scales 14 and at the location of a peak wavelet coefficients continuously increase over scales until the match is optimal. Thus, our scale selection aims at estimating from $\hat{c}(b)$ the scale $a_{\text {dwell }}(b)$ that correlates to the dwell time of a peak. The maximal scale $\hat{a}$ is independently defined for the two wavelets. Since the Haar wavelet matches a narrow local discontinuity it is set to $\hat{a}_{\text {haar }}=20$, corresponding to 2 s time interval. The Bior wavelet covers the whole peak and we therefore define $\hat{a}_{b i o r}=\left(2 \tau f_{s}\right) / r_{\text {bior }}=94$. Here, $\tau$ is the mean dwell time, $r_{b i o r}$ the support range of the Bior wavelet and $f_{s}$ the sampling rate. 


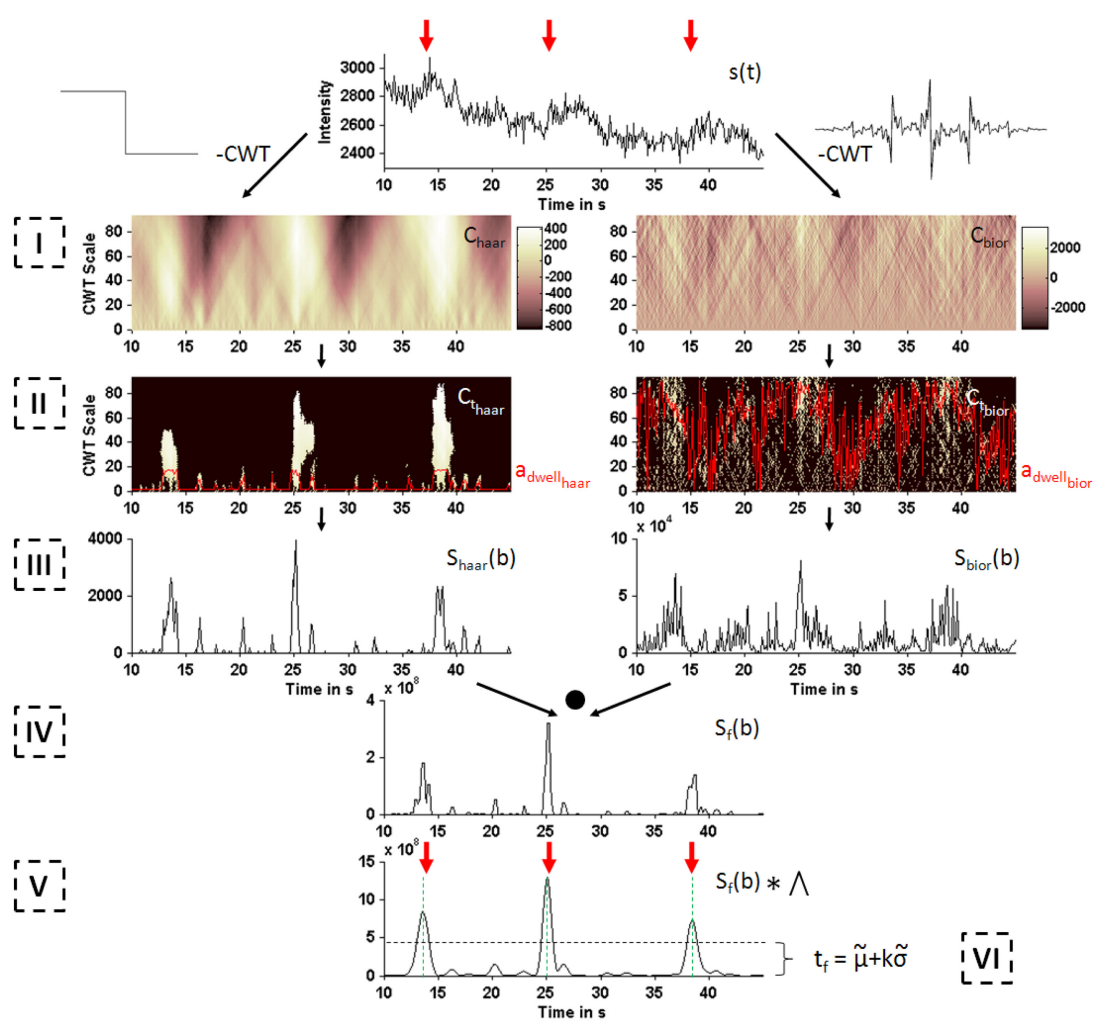

Fig. 3. Steps of MWA: I multiple signal projection into the wavelet space, II selection of relevant coefficients, III combination of coefficients, IV fusion of multi wavelet information, V Bartlett window smoothing and VI peak thresholding

In step III the selected coefficients are combined to the signal $S(b)$ :

$$
S(b)=\sum_{a=1}^{a_{d w e l l}(b)} C_{t}(a, b) .
$$

This is similar to computing the ridge lines in the wavelet coefficients [11] as it also favors continuous scale combinations and tolerates gaps between scales. However, it is computationally much more efficient and requires no additional parameters to be set.

The information from the two wavelets are fused in step IV. In contrast to the multispectra algorithm [15, where Hsueh et al. propose the merging of redundant signals directly in the wavelet coefficients we fuse the complementary signals $S_{\text {haar }}(b)$ and $S_{\text {bior }}(b)$ by pointwise multiplication $(\bullet)$ into $S_{f}(b)$. This can be interpreted as a logical AND operation between two individual peak indications. Further, a third order local maximum filter is applied to each signal before fusion 
to account for the fact that the Haar and Bior wavelet have a location shift at the same peak. Hence, the data fusion becomes:

$$
S_{f}(b)=\max _{b-1 \leq b^{*} \leq b+1} S_{h a a r}\left(b^{*}\right) \bullet \max _{b-1 \leq b^{*} \leq b+1} S_{b i o r}\left(b^{*}\right)
$$

Finally, local maxima are found and compared to a threshold. Therefore in step $\mathrm{V}, S_{f}(b)$ is first smoothed by convolution with the Bartlett window 9 to remove spurious peaks in the vicinity of real peaks as well as plateaus of similar values in $S_{f}(b)$ that can occur due to the maximum filter operation. We finally compute the threshold in step VI using robust statistics: $t_{f}=\tilde{\mu}_{S_{f}}+k \tilde{\sigma}_{S_{f}}$, where $\tilde{\mu}$ is the median and $\tilde{\sigma}$ the median absolute deviation. Hence, under constant imaging conditions, $t_{f}$ is applicable for all synapses without individual adjustment of $k$.

\section{Evaluation}

To evaluate the detection performance of our method, we analyzed real datd 2 that comprises 123 synapse signals from four images acquired at $10 \mathrm{~Hz}$ for $2-3 \mathrm{~min}$. To distinguish between evoked and spontaneous activity, six action potentials have been evoked by single pulse electrical stimulation during each acquisition. Three biological experts individually detected peaks in the data. The inter-rater reliability was surprisingly low. The neurobiologists' ground truths exhibited only a mutual similarity of $34 \pm 10 \%$ (approximated with the Jaccard index) and thus, did not allow a quantitative performance assessment.

As an indirect correctness measure, we instead show in larger data sets that detected peaks exhibit a quantized distribution of peak intensities [2, 4]. As we include off-focus synapses and combine acquisitions from cell preparations with different expression levels, peaks from different synapses have different absolute intensity quanta. Hence, we estimate the individual absolute quantal intensity from the peaks of each synapse by hierarchical clustering. This allows for computing relative peak intensities, which can be combined with other synapses. The distributions for spontaneous activity are shown in Fig. 4a-c. There is only one significant peak in Fig. 4a indicating that all peaks at a synapse originate from the same amount of released vesicles. In comparison, there are several evenly spaced peaks under high $\mathrm{Ca}^{2+}$ concentration suggesting that at single synapses peaks with different intensities did occur. We conclude that correct peaks have been detected, because, as expected, applying high $\mathrm{Ca}^{2+}$ concentration $(5 \mathrm{mM}$ $\mathrm{CaCl}_{2}, 0.6 \mathrm{mM} \mathrm{MgCl} 2$ ) increases the probability for higher numbers of released vesicles per action potential. Analysis of evoked peaks yields equal results.

For a more rigorous test, we applied MWA on synthetic data and compared its performance with amplitude thresholding (AT). The data were created from 60 clearly identifiable evoked and spontaneous peaks that were repeatedly placed on signals of length $3 \mathrm{~min}$. To test the performance over different SNRs, we added

${ }^{2}$ Rat Hippocampal neurons were transfected to couple pHluorin to the vesicle protein vGlut. We performed recordings after 14-18 DIV while perfusing with normal extracellular solution and standard calcium concentration $\left(2 \mathrm{mM} \mathrm{CaCl} 2,2 \mathrm{mM} \mathrm{MgCl}_{2}\right)$. 
scaled noise from real signals that showed no activity. The noise between peaks was also adjusted to match the SNR of the peaks. Similar to the receiver operating characteristic curve, we assess the performance by plotting true positive rate (TPR) and false discovery rate (FDR) for different thresholds $k$ (see Fig. 4 $\mathrm{d}$-e). Clearly, the detection capability of MWA is superior at any SNR for evoked and spontaneous peaks. The relative improvement even increases towards low SNR.

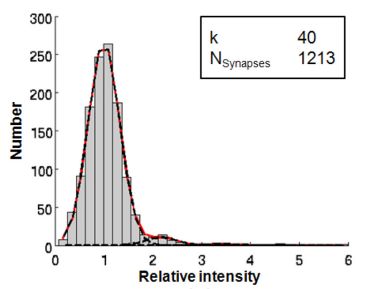

(a)

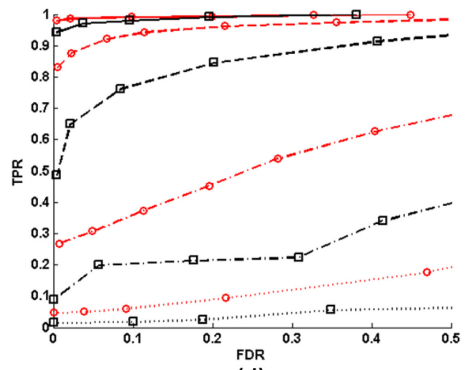

(d)

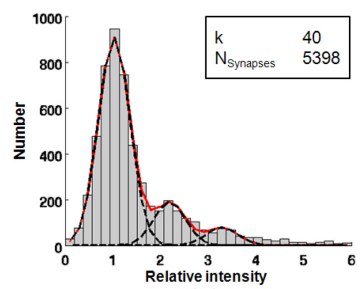

(b)

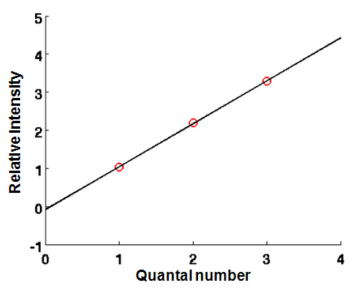

(c)

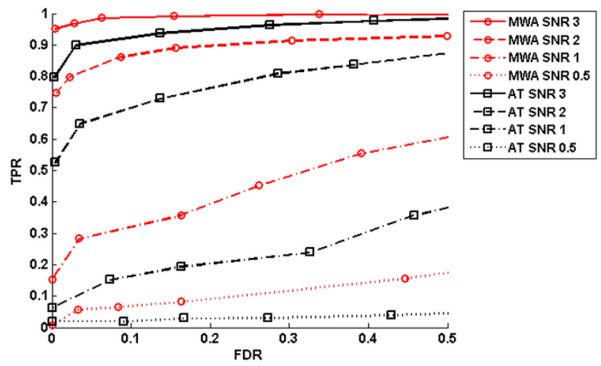

(e)

Fig. 4. Relative intensity distributions for standard (a) and high (b) $\mathrm{Ca}^{2+}$ concentration. The red curve is the overall fit to a set of Gaussians of the same width. The black curve shows the individual Gaussians. The line fit to the fitted peak positions visualizes the integer multiples of the quantal distribution (c). (d) and (e) show the performance assessment over varying SNR for evoked and spontaneous activity, respectively. Each curve is computed from 500 peaks.

\section{Conclusion}

We proposed a multi wavelet algorithm for the improved detection of in particular spontaneous activity that has not been investigated yet. The application of two different wavelets is novel and allows to match the most important peak features. On synthetic data, our method shows superior performance to amplitude thresholding at any SNR and, thus, addresses the requirement for high temporal resolution up to $100 \mathrm{~Hz}$ [3]. On real data, we showed that detected peaks reveal the quantal nature of vesicle release, indicating the correctness of our method. A ground truth evaluation failed due to the poor inter-rater reliability. This underlines the need for automatic approaches. Notably, our method is not limited to two wavelets, but can be used with any number of wavelets, yielding a great potential for the detection of even more varying peak shapes. 
For unsupervised detection, there are still two problems to solve. First, the SNR depends on the size of the synaptic region which itself depends on the setup, particularly the objective and emission wavelength. Instead of computing the intensity signal using a fixed region, 2D Gaussian functions could be fitted to the intensity profile of synapses to track the optimal intensity, which also counteracts motion artefacts. Second, we will investigate the choice of the threshold parameter $k$ more rigorously. In fact, the chosen values $k$ are reasonably robust for imaging synapses in a wider field of view. However, the expression level of cell preparations often varies and an adjustment scheme for $k$ would be preferable.

\section{References}

1. Miesenböck, G., De Angelis, D.A., Rothman, J.E.: Visualizing secretion and synaptic transmission with pH-sensitive green fluorescent proteins. Nature 394, 192-195 (1998)

2. Balaji, J., Ryan, T.A.: Single-vesicle imaging reveals that synaptic vesicle exocytosis and endocytosis are coupled by a single stochastic mode. PNAS 104(51), 20576-20581 (2007)

3. Ariel, P., Ryan, T.A.: Optical mapping of release properties in synapses. Frontiers in Neural Circuits 4(18) (2010)

4. Sinha, R., Ahmed, S., Jahn, R., Klingauf, J.: Two synaptobrevin molecules are sufficient for vesicle fusion in central nervous system synapses. PNAS 108(34), 14318-14323 (2011)

5. Kay, S.M.: Fundamentals of statistical signal processing: detection theory, pp. 94140. Prentice Hall PTR (1998)

6. Jain, A.K., Duin, R.P.W., Mao, J.: Statistical Pattern Recognition: A Review. IEEE Trans. Pattern Anal. Mach. Intell. 22(1), 4-37 (2000)

7. Shahid, S., Walker, J., Smith, L.S.: A New Spike Detection Algorithm for Extracellular Neural Recordings. IEEE Trans. Biomed. Eng. 57(4), 853-866 (2010)

8. Natora, M., Obermayer, K.: An Unsupervised and Drift-Adaptive Spike Detection Algorithm Based on Hybrid Blind Beamforming. EURASIP J. Adv. Signal Process. (2011)

9. Kim, K.H., Kim, S.J.: A Wavelet-Based Method for Action Potential Detection From Extracellular Neural Signal Recording With Low Signal-to-Noise Ratio. IEEE Trans. Biomed. Eng. 50(8), 999-1011 (2003)

10. Benitez, R., Nenadic, Z.: Robust Unsupervised Detection of Action Potentials With Probabilistic Models. IEEE Trans. Biomed. Eng. 55(4), 1344-1354 (2008)

11. Du, P., Kibbe, W.A., Lin, S.M.: Improved Peak Detection in Mass Spectrum by Incorporating Continuous Wavelet Transform-based Pattern Matching. Bioinformatics 22, 2059-2065 (2006)

12. Donoho, D.L., Johnstone, I.M.: Ideal Spatial Adaptation by Wavelet Shrinkage. Biometrika 81, 425-455 (1994)

13. Nenadic, Z., Burdick, J.W.: Spike Detection Using the Continuous Wavelet Transform. IEEE Trans. Biomed. Eng. 52(1), 74-87 (2005)

14. Mallat, S., Zhong, S.: Characterization of Signals from Multiscale Edges. IEEE Trans. Pattern Anal. Mach. Intell. 14(7), 710-732 (1992)

15. Hsueh, H., Kuo, H., Tsai, C.: Multispectra CWT-based algorithm (MCWT) in mass spectra for peak extraction. J. Biopharm. Stat. 18, 869-882 (2008) 